\title{
Health-aware Model Predictive Control of Wind Turbines using Fatigue Prognosis
}

\author{
Hector Sanchez ${ }^{1}$,Teresa Escobet ${ }^{1}$,Vicenç Puig ${ }^{1}$ and Peter Fogh Odgaard ${ }^{2}$ \\ ${ }^{1}$ Automatic Control Department, Universitat Politècnica de Catalunya (UPC), Rambla Sant Nebridi 22, 08222 \\ Terrassa, Spain; (e-mail:\{hector.eloy.sanchez, teresa.escobet, vicenc.puig\}@upc.edu) \\ ${ }^{2}$ Department of Electronic Systems, Automation and Control Section, Aalborg University, Fredrik Bajers Vej 7C, 9220 \\ Aalborg, Denmark; (e-mail:pfo@es.aau.dk)
}

\begin{abstract}
SUMMARY
Wind turbine components are subject to considerable fatigue due to extreme environmental conditions to which are exposed, especially those located offshore. Wind turbine blades are under significant gravitational, inertial and aerodynamic loads which cause fatigue and degradation of this component during the wind turbine operational life. A fatigue problem is often present at the blade root due to the considerable bending moments applied to this zone. The present work investigates the modeling of this phenomenon using the blade root moment information coming from the sensor available in a high fidelity simulator of a utility scale wind turbine. Interest in the integration of control with fatigue load minimization has increased in recent years. The integration of a system health management module with control provides a mechanism for the wind turbine to operate safely and optimize the trade-off between components life and energy production. The research presented in this paper explores the integration of model predictive control (MPC) with fatigue-based prognosis approach to minimize the damage of wind turbine components (the blades). A modified approach based on the rainflow counting algorithm is proposed in order to obtain online information of the blades accumulated damage that can be integrated with MPC. The controller objective function is modified by adding an extra criterion that takes into account the accumulated damage. The scheme is implemented and tested in a well-known wind turbine benchmark. Copyright(C) 2016 John Wiley \& Sons, Ltd.

Received ...
\end{abstract}

KEY WORDS: Prognosis, Fatigue, Wind Turbines, Model Predictive Control

\section{INTRODUCTION}

Wind turbines are subject to a highly irregular loading due to wind, gravity, and aerodynamic effects

which makes them specially vulnerable to fatigue damage. Fatigue as a critical loading pattern has been identified by the scientific community as the cause of majority of structural failures in both composite and conventional structures such as wind turbine blades [1]. Due to the high number of load cycles that occur during the life of the turbine, fatigue considerations are of particular importance in wind turbine control. 
Fatigue can be understood as the breakdown of the material subject to stress, specially when repeated series of stresses are applied. It is a phenomenon that occurs in a microscopic scale, manifesting itself as deterioration or damage. Consequently, it has been widely and exhaustively studied from different perspectives [2]. The awareness of wind turbines as fatigue critical machines has led to a significant amount of research that covers several fatigue analysis techniques for different wind turbine components. A widely damage rule used to formulate fatigue analysis in wind turbines is the Palmgren-Miner linear damage rule [3]. This rule commonly called the Miner's rule is being currently used throughout the industry and in the academia [4] [5]. In addition to Miner's rule based damage analysis, linear crack propagation models had been used for the fatigue analysis of wind turbines with successful applications [6], [7].

The main objective of operational control of wind turbines is to maximize the extracted wind power from the wind. However, wind turbines components are subject to considerable fatigue due to extreme environmental conditions to which are exposed, especially those located offshore. For this reason, interest in the integration of control with fatigue-based prognosis of components has increased in recent years. In the work of [8], wind turbine blade state health information is integrated with contingency controls to mitigate damage in this component. According to [9], in the recent years many advanced control strategies have been proposed for the operational control of wind turbines. Unfortunately, they have not been adopted by the industry. However, the application of model predictive control (MPC) to wind turbines has started to attract the attention of academia and the industry [10] [11] [12], because of the possibility of dealing with the conflicting power optimization and fatigue load reduction problem, as it has been shown in a number of publications. For example, the use of MPC for switching between partial and full load operation of the wind turbine while reducing tower fore-aft fatigue loads was reported in [13]. This paper also addresses pole placement based objective functions, and a discussion of implementation structures for the MPC solution with the existing wind turbine controller as well.

A Full Load Control (FLC) with wind speed predictions based on LIDARS has been proposed in [14]. MPC control for floating and solid foundation wind turbines has been investigated in [15], in this work the application of MPC control for different wind speeds and modes of operation has been addressed. Feedback-Feedforward MPC control applied to the wind turbine collective pitch and torque control problem in full load operation is presented in [16], this work concludes that the use of state constraints in the MPC formulation is useful to avoid unnecessary shutdowns of the wind turbine due to violations of the overspeed limit.

Switch-less control considering tower fore-aft displacement by MPC is the focus of [17] considering a data-driven prediction model. Nonlinear MPC has been used to tackle the nonlinearities in the wind turbine [18] and also has been applied in the work of [19], where LIDAR systems are used to provide information of wind disturbances in various distances in front of the wind turbine. The performance of the proposed nonlinear MPC control is assessed in terms of fatigue loads reduction and power production. A data-based MPC strategy that incorporates fatigue estimation was presented in [20]. In the work of [21], an approach including dynamic inflow into MPC control is proposed to reduce fatigue loads in wind turbine tower.

The research presented in this paper explores a new control paradigm, named "health-aware control" (HAC) that considers the information about the system health to adapt the objectives of the control law to extend the system remaining useful life (RUL) [22]. In this way, the control actions 
are generated to fulfill the control objectives/constraints but at the same time to extend the life of the system components. So, HAC tries to achieve maximum performance while not degrading the system so much. In case that the controller is implemented using MPC, the trade-off is based on modifying the control objective function including new terms that take care of the system health and leading solve a multi-objective optimization problem where a trade-off between system health and performance should be established [23].

The main contribution of this paper relies on the development of HAC approach based on MPC with application to wind turbines. This approach integrates fatigue-based prognosis with the aim of minimizing the damage of wind turbine components (the blades) while still maximizing the extracted wind power from the wind. The integration of a system health management module with MPC control is done by developing a fatigue-based model using the rain-flow counting approach and adding an extra criterion in the control objective function that takes into account the accumulated damage. This provides the wind turbine a mechanism to operate safely and optimize the trade-off between components life and energy production. The proposed approach is implemented and tested using a high fidelity simulator of a utility scale wind turbine called FAST (Fatigue, Aerodynamics, Structures, and Turbulence), which is a comprehensive aeroelastic simulator capable of predicting both the extreme and fatigue loads of two and three bladed horizontal-axis wind turbines (HAWTs) developed by NREL (National Renewable Energy Laboratory) in Colorado, USA [24]. The wind turbine simulator has been used by the FDI/FTC community and has been proposed for Fault Diagnosis and Fault Tolerant Control of a wind turbine benchmark [25].

The remainder of the paper is organized as follows. Section 2 presents the application of MPC to wind turbines. Section 3 introduces the fatigue analysis applied to wind turbines and describes how to implement health-aware control using MPC to wind turbines. Section 4 describes the case study based on the wind turbine benchmark, where the proposed approach is assessed and the results are analyzed. Section 5 highlights the concluding remarks and some future research directions.

\section{MPC OF WIND TURBINES}

In order to use MPC, a model of the system is necessary. In this section, a nonlinear model for the wind turbine collected from the literature is presented and subsequently a linear model to be used for the MPC is obtained around a generic operating point.

\subsection{Wind turbine model}

The considered wind turbine model, used as a basis for prediction model in an MPC scheme, is created taking as reference the one used in [21]. This wind turbine is simulated using FAST from NREL which is a high fidelity aero elastic wind turbine model, see [24] and it is based on the $5 \mathrm{MW}$ NREL reference turbine, see [26].

The wind turbine model is created from the dynamic behavior equations of each of the subsystems: aerodynamic part, drive train, tower, pitch actuator and electric generator. The overall aerodynamic model can be summarized by the following nonlinear equations [27]: 


$$
\begin{aligned}
& T_{a}(t)=\frac{1}{2 \omega_{r}(t)} \rho A v_{a}^{3}(t) C_{p}(\lambda(t), \beta(t)), \\
& F_{w}(t)=\frac{1}{2 \omega_{r}(t)} \rho A v_{a}^{3}(t) C_{t}(\lambda(t), \beta(t)),
\end{aligned}
$$

where $T_{a}$ is the rotor torque, $F_{w}(t)$ is the force acting on the tower, $\omega_{r}(t)$ is the shatf's rotational speed, $v_{a}(t)$ is the wind speed experienced at the rotor, $\rho$ is the air density, $A$ is the swept area, $C_{p}(\cdot)$ is the power coefficient and $C_{t}(\cdot)$ is the thrust coefficient. The power and the thrust coefficients are functions of the pitch angle of the blades, $\beta(t)$, and the tip-speed ratio, $\lambda(t)$, which is defined as

$$
\lambda(t):=\frac{R \omega_{r}(t)}{v_{a}(t)}
$$

where $R$ is the blades' radius. The wind speed experienced at the rotor, $v_{a}$ (that depends of the wind speed, $v_{w}$, and the tower fore-aft movements $v_{t}$ ) can be computed as:

$$
v_{a}(t)=v_{w}(t)-v_{t}(t)
$$

according to [27].

The drive train can be modeled as a simple one mass system, without any friction and energy losses and omitting the drive train torsion. All the inertias in the system (inertia of the rotor, gearbox, low and high-speed shaft) are lumped into one inertia, $J$. The dynamics of the drive train can thereby be described as:

$$
\dot{\omega}_{r}(t)=\frac{T_{a}(t)-N T_{g}(t)}{J},
$$

where $T_{g}(t)$ is the torque applied by the generator and $N$ is the gear ratio. The generator angular speed, $\omega_{g}$, is found as:

$$
\omega_{g}(t)=N \omega_{r}(t)
$$

The tower system is modeled as the effect which causes a disturbance input, the wind, on the deflection of the tower as in [28]. The model is simplified to a mass, spring, damper system where $M_{t}$ covers the lumped mass of the tower, rotor and nacelle, considering negligible the tower oscillations. The forces acting on the mass comes from the damper, spring and the disturbance input from the wind:

$$
a_{t}(t) M_{t}=-B_{t} v_{t}(t)-K_{t} d_{t}(t)+F_{w}(t)
$$

where: $a_{t}$ is the acceleration of the lumped mass, $B_{t}$ is the friction coefficient, $K_{t}$ is the spring coefficient, $M_{t}$ is the mass of the lumped mass and $d_{t}$ is the tower fore-aft displacement.

The pitch actuators on a wind turbine controls the pitch of the blades. Usually, the pitch system can be hydraulic or electrical. Compared with pitch system, other subsystems have a slower response time. The the response of the actuators is modeled by means of a first order system as in [27]:

$$
\dot{\beta}(t)=\frac{1}{\tau_{p}} \beta_{r e f}(t)-\frac{1}{\tau_{p}} \beta(t),
$$


where $\beta_{r e f}(t)$ is the reference signal for the pitch angle, $\beta(t)$ is the pitch angle and $\tau_{p}$ is the time constant.

Electric power is generated by the generator, while a power converter interfaces the wind turbine generator output with the utility grid and controls the currents in the generator. As in pitch subsystem, the dynamics of the generator torque system is assumed to be much faster, than of the rest of the control system. Finally, the converter dynamics are approximated by a first order system with time constant $\tau_{g}$ as in [29]. The continuous time model of the generator system is:

$$
\dot{T}_{g}(t)=\frac{1}{\tau_{g}} T_{g, r e f}(t)-\frac{1}{\tau_{g}} T_{g}(t),
$$

where $T_{g}(t)$ is the generator torque and $T_{g, r e f}(t)$ is the reference for the generator torque. The power output of the generator, $P_{g}$, is described as:

$$
P_{g}(t)=\eta_{g} T_{g}(t) \omega_{g}(t)
$$

where $\eta_{g}$ is the generator efficiency.

The component models can now be gathered into a nonlinear state space model, which is used by the MPC after linearization. The state equations are as follows

$$
\left[\begin{array}{c}
\dot{\omega}_{r} \\
\dot{d}_{t} \\
\dot{v}_{t} \\
\dot{\beta} \\
\dot{T}_{g}
\end{array}\right]=\left[\begin{array}{c}
\frac{1}{J}\left(T_{a}-N T_{g}\right) \\
v_{t} \\
\frac{1}{M_{t}}\left(F_{w}-K_{t} d_{t}-B_{t} v_{t}\right) \\
\frac{1}{\tau_{p}}\left(\beta_{r e f}-\beta\right) \\
\frac{1}{\tau_{p}}\left(T_{g, r e f}-T_{g}\right)
\end{array}\right]
$$

while the output equations are given by:

$$
\left[\begin{array}{c}
P_{g, m} \\
v_{t, m} \\
\omega_{r, m}
\end{array}\right]=\left[\begin{array}{c}
\eta_{g} N \omega_{r} T_{g} \\
v_{t} \\
\omega_{r}
\end{array}\right]
$$

\subsection{Control-oriented model}

Control of wind turbines deal with a number of objectives and tasks. Many of these are conflicting in nature, in this work a subset of these objectives are considered. The idea is to design an MPC which deals with the conflicting objectives of generating nominal power while minimizing the blade root fatigue state and the tower fore-aft fatigue loads, when operating the wind turbine at above rated wind speed.

In order to have a model that can be used with an MPC controller, the nonlinear model needs to be linearized.

The linearized model is derived from (11) and (12) applying the linearization procedure based on Taylor series expansion at a particular operating point.

Given the nonlinear system described by (11) and (12), an equilibrium point of the states, denoted as $x^{*}$, obtained when the inputs $u=u^{*}$ and disturbances $w=w^{*}$, the linear discrete-time state space representation have the form: 


$$
\begin{aligned}
x(k+1) & =A x(k)+B u(k)+E w(k), \\
y(k) & =C x(k),
\end{aligned}
$$

The model includes states representing the rotor speed $\omega_{r}$, the tower fore-aft displacement $d_{t}$, the tower fore-aft velocity $v_{t}$, the generator torque $T_{g}$ and the pitch angle $\beta$. The controlled inputs are generator torque and pitch references, respectively $T_{g, r e f}$ and $\beta_{r e f}$. The wind speed $v_{w}$ is a measured non-controlled input. The model outputs are the generated power $P_{g}$, the tower fore-aft velocity $v_{t}$ and the rotor speed $\omega_{r}$. The new inputs $u$, disturbances $w$, states $x$, and outputs $y$ represent the variation of $u, y, w$ and $x$ from the equilibrium value, and are described below in equations (15)-(18).

$$
\begin{aligned}
& u=\left[\begin{array}{ll}
T_{g, r e f}-T_{g, r e f}^{*} & \beta_{r e f}-\beta_{r e f}^{*}
\end{array}\right]^{T}, \\
& w=\left[v_{w}-v_{w}^{*}\right] \text {, } \\
& x=\left[\begin{array}{lllll}
\omega_{r}-\omega_{r}^{*} & d_{t}-d_{t}^{*} & v_{t}-v_{t}^{*} & T_{g}-T_{g}^{*} & \beta-\beta^{*}
\end{array}\right]^{T}, \\
& y=\left[\begin{array}{lll}
P_{g, m}-P_{g}^{*} & v_{t, m}-v_{t}^{*} & \omega_{r, m}-\omega_{r}^{*}
\end{array}\right]^{T} \text {. }
\end{aligned}
$$

And the state space matrix $A$, input matrix $B$, disturbance matrix $E$ and output matrix $C$ are

$$
\begin{gathered}
A=\left[\begin{array}{ccccc}
1+\frac{T_{s}}{J} \frac{\partial T_{a}}{\partial \omega_{r}} & 0 & -\frac{T_{s}}{J} \frac{\partial T_{a}}{\partial v_{t}} & \frac{T_{s}}{J} \frac{\partial T_{a}}{\partial \beta} & -\frac{N}{J} \\
0 & 1+T_{s} & 0 & 0 & 0 \\
\frac{T_{s}}{M_{t}} \frac{\partial F_{w}}{\partial \omega_{r}} & -\frac{K_{t} T_{s}}{M_{t}} & 1+\frac{T_{s}}{M_{t}}\left(-B_{t}-\frac{\partial F_{w}}{\partial v_{t}}\right) & \frac{T_{s}}{M_{t}} \frac{\partial F_{w}}{\partial \beta} & 0 \\
0 & 0 & 0 & 1-\frac{T_{s}}{\tau_{p}} & 0 \\
0 & 0 & 0 & 0 & 1-\frac{T_{s}}{\tau_{g}}
\end{array}\right] \\
B=\left[\begin{array}{ccc}
0 & 0 \\
0 & 0 \\
0 & 0 \\
0 & \frac{T_{s}}{\tau_{p}} \\
\frac{T_{s}}{\tau_{g}} & 0
\end{array}\right] \\
E=\left[\begin{array}{ccccc}
\frac{T_{s}}{J} \frac{\partial T_{a}}{\partial v_{\omega}} & 0 & \frac{T_{s}}{M_{t}} \frac{\partial F_{w}}{\partial v_{\omega}} & 0 & 0
\end{array}\right] \\
C=\left[\begin{array}{ccccc}
\frac{\partial P_{g}}{\partial \omega_{r}} & 0 & 0 & 0 & \frac{\partial P_{g}}{\partial T_{g}} \\
0 & 0 & 1 & 0 & 0 \\
1 & 0 & 0 & 0 & 0
\end{array}\right]
\end{gathered}
$$

where $T_{s}$ is the sampling time. 


\subsection{Standard $M P C$}

MPC uses a mathematical model to calculate the optimal control actions according to a given cost function [30]. In this paper, it is assumed that the system behavior can be described at each time instant $k \in \mathbb{Z}$ by the discrete-time model (13).

It is also considered that the system is subject to state and input constraints, which can be posed as

$$
\begin{aligned}
& x(k) \in \mathbb{X} \triangleq\left\{x(k) \in \mathbb{R}^{n_{x}} \mid \underline{x} \leq x(k) \leq \bar{x}, \forall k\right\}, \\
& u(k) \in \mathbb{U} \triangleq\left\{u(k) \in \mathbb{R}^{n^{u}} \mid \underline{u} \leq u(k) \leq \bar{u}, \forall k\right\},
\end{aligned}
$$

The control goal is to minimize a convex (possible multi-objective) cost function $\quad \ell(x, u): \mathbb{X} \times \mathbb{U} \rightarrow \mathbb{R}$, which might bear any functional relationship to the operating cost of the system. From the model in (13), let $\hat{w}\left(k: k+H_{p}-1\right) \triangleq\left(\hat{w}(k), \hat{w}(k+1 \mid k), \ldots, \hat{w}\left(k+H_{p}-1 \mid k\right)\right)$ be the sequence of disturbances over a fixed time prediction horizon $H_{p} \in \mathbb{Z}_{+}$. The first element of the sequence is measured, while the rest of the elements, i.e., $\hat{w}(k+i \mid k)$, denote estimates of future disturbances computed by an exogenous system and available at each time instant $k$. Hence, the MPC controller design is based on the solution of the following finite horizon optimization problem (FHOP):

$$
\min _{\mathbf{u}_{k}} \sum_{i=0}^{H_{p}-1}\left[\|e(k+i \mid k)\|_{W_{e}}^{2}+\|u(k+i \mid k)\|_{W_{u}}^{2}+\|\Delta u(k+i \mid k)\|_{W_{\Delta u}}^{2}\right],
$$

subject to

$$
\begin{aligned}
& x(k+i+1 \mid k)=A x(k+i \mid k)+B u(k+i \mid k)+E \hat{w}(k+i \mid k), \\
& e(k+i+1 \mid k)=r(k+i+1 \mid k)-C x(k+i \mid k), \\
& \Delta u(k+i \mid k)=u(k+i \mid k)-u(k+i-1 \mid k), \\
& u(k+i \mid k) \in \mathbb{U}, \\
& x(k+i \mid k) \in \mathbb{X}, \\
& (x(k \mid k), u(k-1 \mid k), \hat{w}(k \mid k))=\left(x_{k}, u_{k-1}, w_{k}\right),
\end{aligned}
$$

for all $i \in \mathbb{Z}_{\left[0, H_{p}-1\right]}$, where $\mathbf{u}_{k}=\left\{u_{k+i \mid k}\right\}_{i \in \mathbb{Z}_{\left[0, H_{p}-1\right]}}$ are the decision variables, with $\mathbf{u}_{k}$ being the sequence of controlled inputs. Moreover, $r_{k+i \mid k}$ are the set-points for the controlled variables and $\hat{w}_{k+i \mid k}$ are the forecasted disturbances for the $i$-step ahead from $k$. Weighting matrices $W_{e} \in \mathbb{R}_{+}^{n_{y} \times n_{y}}$, $W_{u} \in \mathbb{R}_{+}^{n_{u} \times n_{u}}$ and $W_{\Delta u} \in \mathbb{R}_{+}^{n_{u} \times n_{u}}$ are used to establish the priority of the different control objectives, that are, tracking error, control effort and smoothness, respectively. Constraint $(24 \mathrm{~g})$ represents the measurements available at time step $k$.

From the optimal solution of (24) $\mathbf{u}_{k}^{\star}$ at time step $k$, only the first optimal control action is applied, i.e., $u_{k}=u_{k \mid k}^{\star}$, following the MPC receding horizon philosophy. Then, the new measurements are collected to initialize initial conditions (24g) and the optimization problem (24) is solved again. This procedure is repeated at each time step $k$. 


\section{HEALTH-AWARE MPC}

In this section, the rainflow counting algorithm is described to later on formulate the application and integration with MPC control for the wind turbine case study. The wind turbine stress information is included in model used by the MPC law as an additional state of the system.

\subsection{Rainflow counting algorithm}

Fatigue is the damage accumulation process on a component produced by cyclic loading. Exposing a material to cyclic loading of constant amplitude will cause fatigue failure after a certain number of cycles. In reality amplitudes of cyclic loading are rarely constant. Most components are exposed to random load fluctuations. A common method to quantify the fatigue impact of fluctuating loads is the combination of a rainflow counting (RFC) algorithm and a damage equivalent load approach, enabling the relative comparison of different load samples [31].

RFC method, first introduced by [32], has a complex sequential and nonlinear structure in order to decompose arbitrary sequences of loads into cycles. The rainflow cycle distributions (often simply called cycle distributions or rainflow spectra) represent the occurrence probability of load cycles with different ranges. Usually, to compute a lifetime estimate from a given stress input signal, the RFC method is applied by counting cycles and maxima, jointly with the Palmgren-Miner rule to calculate the expected damage. The input signal is obtained from time history of the loading parameter of interest, such as force, torque, stress, strain, acceleration, or deflection [33]. The Fig. 1 depicts the described procedure.

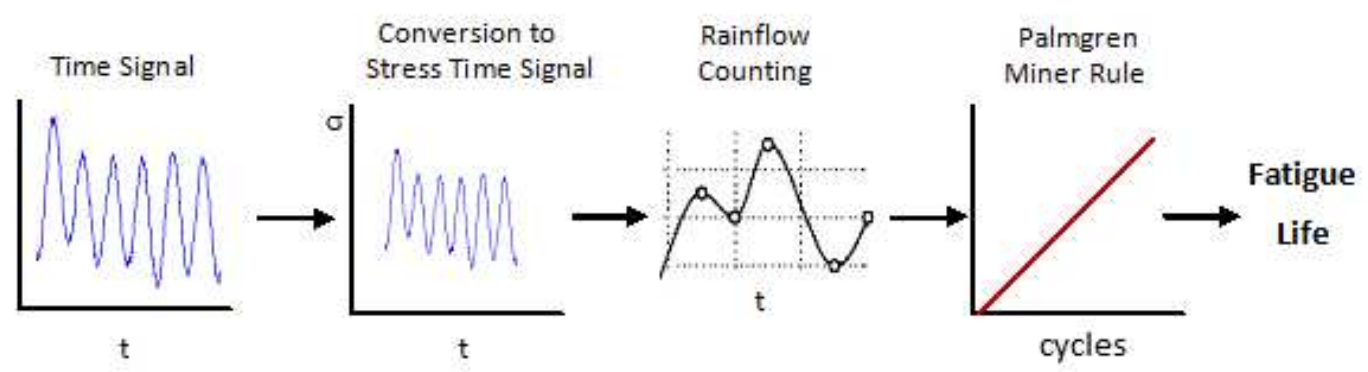

Figure 1. Rainflow counting damage estimation procedure

Different types of RFC algorithms have been proposed in the literature [34][35]. The algorithm used in this paper is introduced in [36], and is implemented as a Matlab code. This algorithm calculates the stress for each rainflow cycle in four steps:

- the stress history is converted to an extremum sequence of alternating maxima and minima;

- for each local maximum $M_{j}$, the left and right region where all stress values are below $M_{j}$ is identified, denoted respectively as $m_{j}^{-}$and $m_{j}^{+}$;

- the minimum stress value is computed being $m_{j}=\min \left\{m_{j}^{-}, m_{j}^{+}\right\}$

- the equivalent stress per rainflow cycle $s_{j}$ associated with $M_{j}$ is given by the amplitude $s_{j}=M_{j}-m_{j}$ or the mean value $s_{j}=\frac{M_{j}+m_{j}}{2}$. 
The damage, $D$, at each stress cycle is computed using S-N curve [37]. The $\mathrm{S}-\mathrm{N}$ curve is a graphical representation of the stress, $s$, versus the number of stress cycles, $N$. An often-used model for the $\mathrm{S}-\mathrm{N}$ curve is

$$
s^{c_{W}} N=K,
$$

where the quantities $K$ and $c_{W}$ are material properties, being $c_{W}$ the Wöhler-coefficient. The damage imposed by a stress cycle with a range $s_{j}$ is computed as

$$
D_{j} \equiv \frac{1}{N_{j}}=\frac{1}{K} s_{j}^{c_{W}}
$$

The accumulated damage after $N$ cycles can be computed using the Palmgren-Miner's damage rule, given by

$$
D_{a c}=\sum_{j=1}^{N} \frac{1}{K} s_{j}^{c_{W}}
$$

The algorithm steps are illustrated with an example shown in Fig. 2. On the top left (a.1), the time signal of the input stress is shown, then the signal is converted into a sequence of maxima and minima (turning points) shown in (a.2). In the bottom part (b.1) it is shown the calculated damage for each rainflow cycle individually. Finally, the accumulated damage is shown on part (b.2).
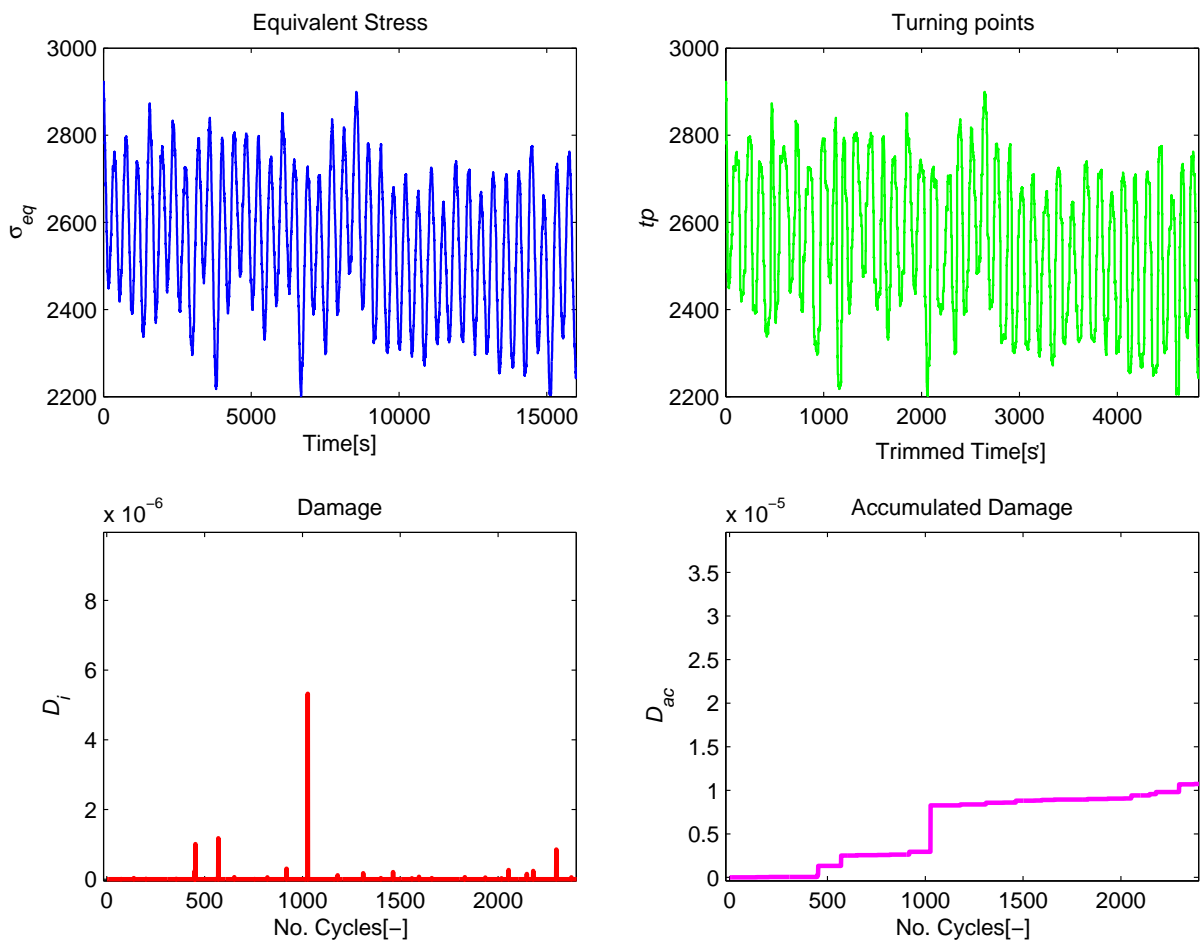

Figure 2. Example of the application of rainflow counting procedure on a blade root moment stress signal obtained from FAST 


\subsection{Application to the wind turbine}

For real-time applications, applying the traditional rainflow counting algorithm is very challenging and computationally heavy. Significant amounts of data must be stored and processed periodically to obtain a magnitude of the data in equivalent regular cycles. In addition, the algorithm must be applied to a stored set of data. To address this challenge, an approach based on the rainflow counting algorithm that can operate in real time has been proposed. In previous works, efficiency of rainflow counting implementations have been proposed, see for example [2].

Loads in wind turbine structure arise from several factors [38], being the main cause the spatial variations of wind speed caused by the turbulent nature of wind. This spatial difference in wind speeds upon blades results in different loading of the wind turbine blades depending on their intermittent position. The paper [38] concludes that the most pronounced contribution to the blade root loading happens at the frequency given by the blades speed, and this loading is the main source of fatigue at the blades and the hub. In case that the input signal is expressed as bending moments, it is necessary to convert the fatigue load to fatigue stress dividing by the appropriate section modulus [39].

Using the RFC method, the accumulated damage is obtained as function of the cycles of the blade root moment stress signal. In order to have available an accumulated damage variable that can be integrated with a linear MPC model, a simplified approach to calculate fatigue on a time series signal is proposed based on RFC theory explained in Section 3.1. The result of this approach is that the accumulated damage is obtained as a function of time instead of the number of cycles. The proposed approach detects the changes of sign which corresponds to a cycle in the stress time signal. The obtained function at each sample step $k$ is the following:

$$
D(k)=\left\{\begin{array}{llc}
0 & \text { if } & I(k)=I(k-1) \\
\frac{1}{K}(s(k))^{c_{W}} & \text { if } & I(k) \neq I(k-1)
\end{array}\right.
$$

where $s(k)$ is the stress at time $k$

$$
s(k)=\frac{1}{L} \sum_{p=k-L}^{k} M_{B, i}(p),
$$

$I(k)$ is the signal adapted to detect cycles (30)

$$
I(k)=M_{B, i}(k)-s(k)
$$

$L$ is the number of samples per cycle and $M_{B, i}$ is the blade root moment of blade $i$.

The accumulated damage is calculated as follows

$$
\hat{D}_{a c c}(k)=\hat{D}_{a c c}(k-1)+D(k)
$$

In this paper, the RFC method applied to the wind turbines considers the blade root moment as the time stress signal in (28). Fig. 3 shows the accumulated damage value obtained with the RFC method and by menas of (31). Notice that at the end of the scenario the accumulated damage is almost the same, the difference as explained before relies on the fact that the damage obtained by 
RFC method is expressed as a function of the cycles count while the method that uses 3 considers the damage as a function of time.
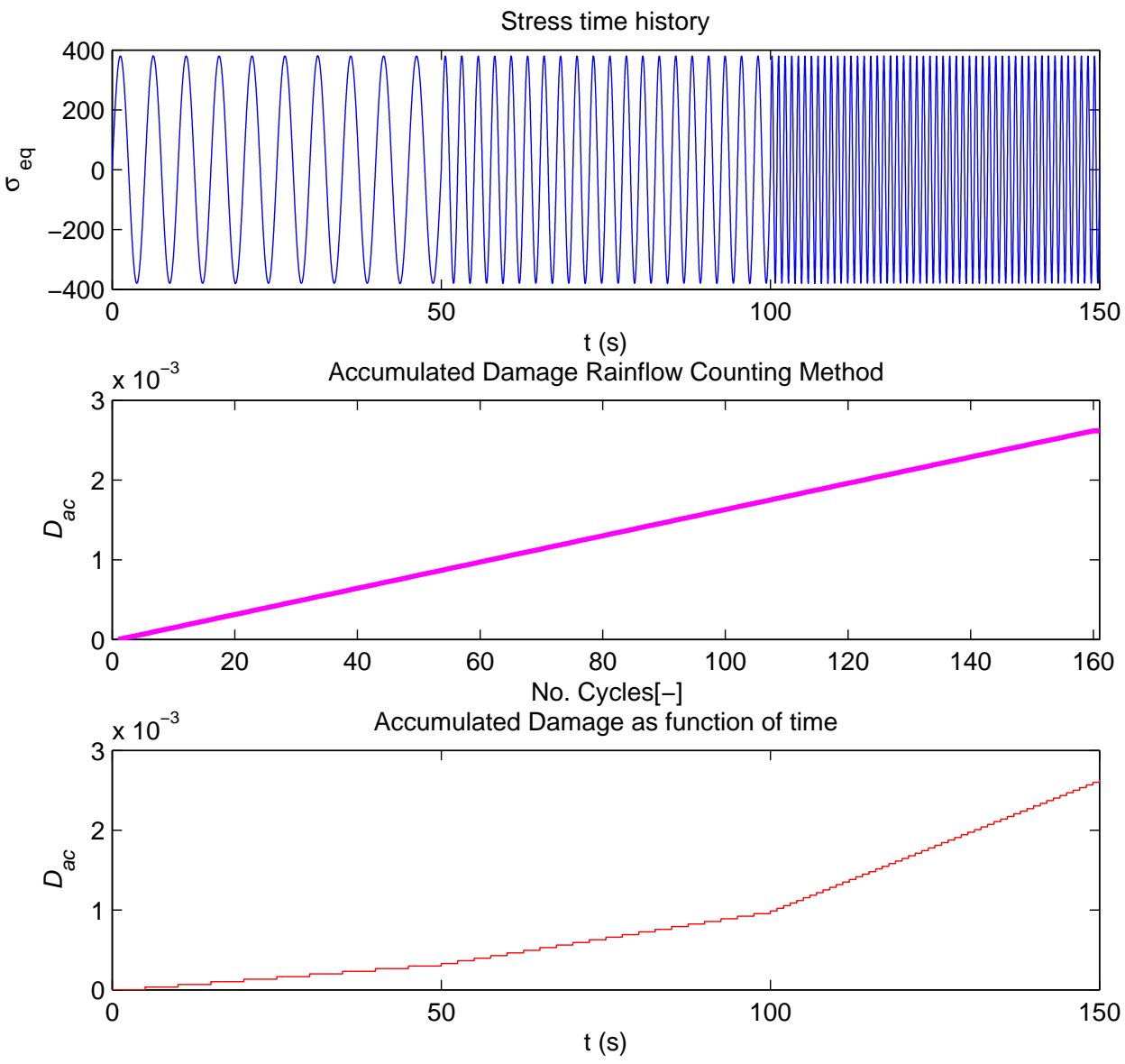

Figure 3. Accumulated Damage Comparison

\subsection{Health-aware $M P C$}

As described in Section 3.2, the degradation process of the wind turbine blade can be evaluated using the blade root moment sensor information. In order to include a new objective in the MPC that aims to reduce the accumulated damage, the RFC model is approximated by means of a linear model.

As a first approximation, after observing that the proposed approach gives a very close approximation of the accumulated damage obtained by the RFC method (Figure 3), the slope $m$ of the accumulated damage curve in function of time is calculated. This function is approximated by means of a linear fatigue-damage model.

In a preliminary work [40], after conducting several tests performed on the wind turbine benchmark implemented in FAST simulator, an experimental model that relates the mean values 
of the blade root moment and pitch angle signals in steady state was proposed. Figure 4 shows the relation between the mean blade root moment as function of the pitch angle considering a first, second and third order polynomials. Finally, the proposed model for the blade root moment dynamics is a first order blade root moment mean model with an slope of $a_{1}$ plus a constant value $a_{0}$ as follows

$$
\bar{M}_{B, i}(k)=a_{1} \beta_{i}(k)+a_{0}
$$

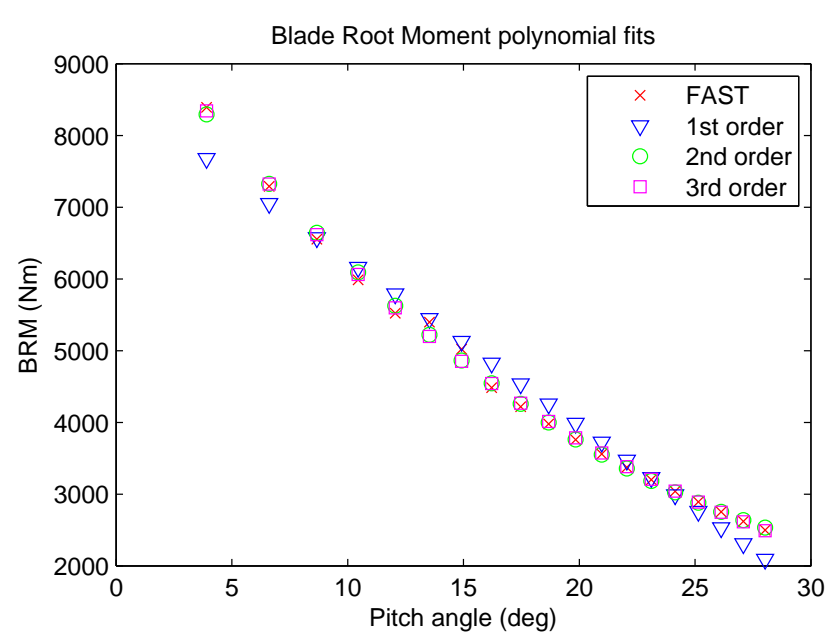

Figure 4. Blade root moments models

Assuming a cycle with a constant wind speed $w_{r}$ and knowing the sampling time $T_{s}$, the number of samples per cycle $L$ can be determined. The proposed linear fatigue-damage model is a function of the pitch value signal which establishes a relation between the control signal and the accumulated damage of the blade root moment:

$$
z(k+1)=z(k)+\frac{m}{L}\left(a_{1} \beta_{i}(k)+a_{0}\right)
$$

where $z(k+1)$ is the accumulated damage of the blade root moment. Equation (33) can be included in the MPC as a new state and additional objective is added in the MPC cost function (24a) to minimize the accumulated damage. The inclusion of the new state $z(k)$ in the state space model (13) leads to an augmented state space representation that replaces (24b) and (24c) in the MPC optimization problem (24). Figure 5 presents the fitting between the RFC approximation as a function of time presented in Section 3.2 and the linear $z$ state damage model introduced in (33). According to [1], it is expected that a wind turbine blade would be required to sustain $10^{9}$ fatigue cycles in 25 years of operational life. In Figure 5 is shown the accumulated damage for a time frame that is of interest for the MPC controller in terms of the prediction and control horizon, the slope of the curves shown would be maintained until the end of the fatigue life if the wind turbine continues to operate with the loads for the wind scenario considered in the simulation. 


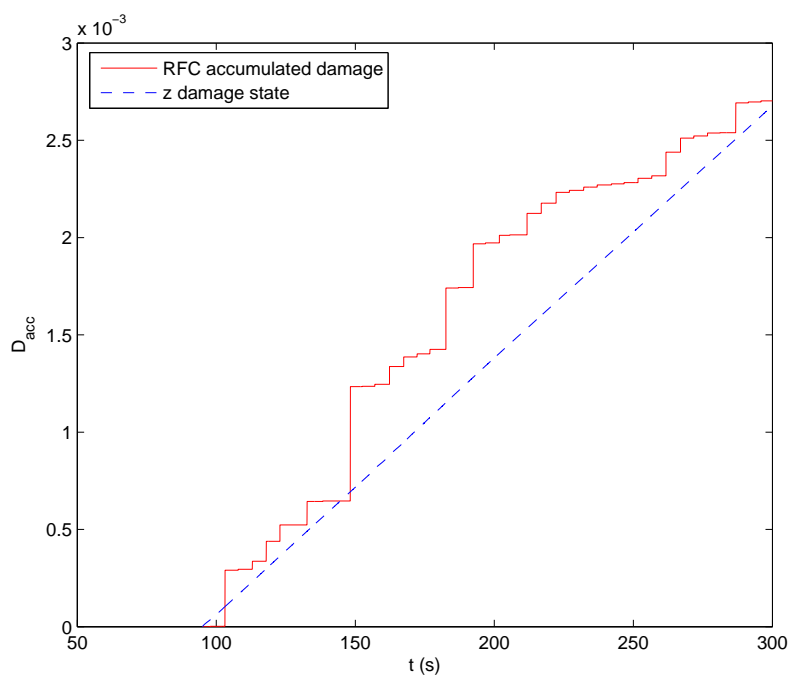

Figure 5. Accumulated damage RFC as a function of time and $z$ fatigue damage state

Taking into account (33), the MPC problem (34) can be formulated as follows:

$$
\min _{\mathbf{u}_{k}} \sum_{i=0}^{H_{p}-1}\left[\|e(k+i \mid k)\|_{W_{e}}^{2}+\|u(k+i \mid k)\|_{W_{u}}^{2}+\|\Delta u(k+i \mid k)\|_{W_{\Delta u}}^{2}+\|z(k+i \mid k)\|_{W_{z}}^{2}\right],
$$

subject to

$$
\begin{aligned}
& x(k+i+1 \mid k)=A x(k+i \mid k)+B u(k+i \mid k)+E \hat{w}(k+i \mid k), \\
& e(k+i+1 \mid k)=r(k+i+1 \mid k)-C x(k+i \mid k), \\
& z(k+1)=z(k)+\frac{m}{L}\left(a_{1} \beta_{i}(k)+a_{0}\right) \\
& \Delta u(k+i \mid k)=u(k+i \mid k)-u(k+i-1 \mid k), \\
& u(k+i \mid k) \in \mathbb{U}, \\
& x(k+i \mid k) \in \mathbb{X}, \\
& (x(k \mid k), u(k-1 \mid k), \hat{w}(k \mid k))=\left(x_{k}, u_{k-1}, \hat{w}_{k}\right),
\end{aligned}
$$

where an additional objective with the corresponding weight $W_{z}$ is added in the MPC cost function (24a) to minimize the accumulated damage.

\section{CASE STUDY}

\subsection{Benchmark description}

The case study used to illustrate the approach proposed in this paper is based on the wind turbine benchmark model introduced in [25]. The wind turbine model is implemented in FAST simulator and it is based on a $5 \mathrm{MW}$ three bladed variable speed wind turbine developed by NREL for 
scientific research*. This model has been used to establish the reference specifications for a number of research projects supported by the U.S. DOEs Wind and Hydropower Technologies Program, the integrated European Union UpWind research program and the International Energy Agency (IEA).

The NREL 5 MW model has been, used as a reference by research teams throughout the world to standardize baseline offshore wind turbine specifications and to quantify the benefits of advanced land and sea-based wind energy technologies. The turbine's hub height is $89.6 \mathrm{~m}$ and the rotor radius is $63 \mathrm{~m}$ with a rated rotor speed is $12.1 \mathrm{rpm}$ while the generator speed is $1200 \mathrm{rpm}$. The simulator also include baseline controllers that allow to control the three pitch angles, generator and converter torques and yaw position. Different measurements are available from sensors as well as the control references.

Figure 6 presents a block diagram of the wind turbine simulation model, provided with the benchmark, including the feedback loops corresponding to the pitch, yaw and torque variables. In this figure, it is also shown the fatigue model block that includes a more realistic fatigue model which provides a more accurate estimate that can be used to feedback the MPC controller in a determined interval of time in order to correct the drifting/biasing estimates of the simplified fatigue model.

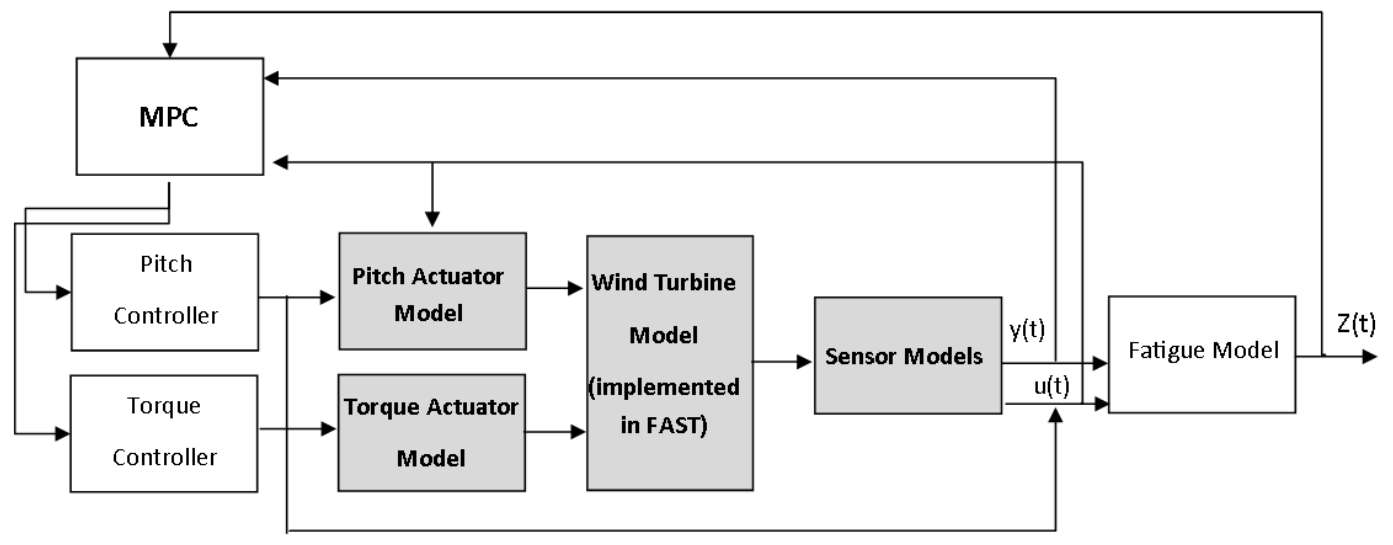

Figure 6. Block diagram of wind turbine simulation and fatigue model

\subsection{Implementation of MPC with health-aware objective}

The health-aware MPC is implemented using Matlab MPC toolbox considering the wind turbine linear model introduced in Section 2.2 and adding the accumulated damage model (33) presented in Section 3 , as a new state

$$
\begin{aligned}
x(k+1) & =A_{d} x(k)+B_{d} u(k)+E_{d} w(k), \\
y(k) & =C_{d} x(k) .
\end{aligned}
$$


where the state and output vector are given by $x_{d}=[x z]^{T}$ and $y_{d}=[y z]^{T}$ respectively. The new matrices are defined as

$$
\begin{gathered}
A_{d}=\left[\begin{array}{cc}
A & 0 \\
0 & \frac{a_{0} m}{L T_{s}}
\end{array}\right] \\
B_{d}=B \\
C_{d}=\left[\begin{array}{ll}
C & 0 \\
0 & 1
\end{array}\right] \\
E_{d}=\left[\begin{array}{cc}
E & 0 \\
0 & \frac{m}{L T_{s}}
\end{array}\right]
\end{gathered}
$$

where $A, B, C$ and $E$ are the linear state space matrices of the wind turbine model defined in (19)(22).

MPC has been implemented using prediction horizon $H_{p}=200$ with a sampling time of $T_{s}=$ $0.0125 \mathrm{~s}$. The MPC objective function (34a) contains the following objectives:

- to track the reference power $P_{g, r e f}$ and rotor speed $\omega_{r, \text { ref }}$ while the tower fore-aft movements are minimized.

- the accumulated damaged evaluated as (33) is minimized.

\subsection{Results}

The health-aware MPC controller results are presented when the wind turbine is operating in the pitch control region (region 3) for several wind speeds and varying the weight of associated to the blade health $W_{z}$.

Figure 7 presents the evolution of the accumulated damages for a turbulent wind of $14 \mathrm{~m} / \mathrm{s}$ mean speed and the performance of the system assessed in terms of different wind turbine variables such as the rotor speed, the pitch angle and the generated power. It can be observed that the inclusion of the fatigue objective mitigates the accumulated damage (assessed with the blade root moment and the rain-flow counting approach). Figure 8 shows the evolution of the blade health state $z$ in more detail. It can be observed that during the transients (see Figure 8.a), more damage is accumulated depending on the weight assigned to the health term $\left(W_{z}\right)$ of the MPC controller (see Figure 8.b). But, on the other hand, the energy extracted from the wind (accumulated extracted power) is reduced. It can also be observed that the MPC controller reduces the rotor speed derating the wind turbine to a lower generated power. This shows the trade-off between maximizing the extracted power and minimizing the accumulated damage in the blades. It is an open research topic to find the best trade-off between maximum power while reducing the accumulated damage.

Tables I and II summarizes the values of the different wind turbine variables altogether with the blade health state $z$ for different values of the health weight $W_{z}(1,10$ and 15) of the MPC controller when two different wind speed scenarios $(14 \mathrm{~m} / \mathrm{s}$ and $17 \mathrm{~m} / \mathrm{s})$ are analysed. From this table, in can be observed that increasing the value of weight $W_{z}$ the accumulated damage is reduced but at the price of decreasing the generated power. 

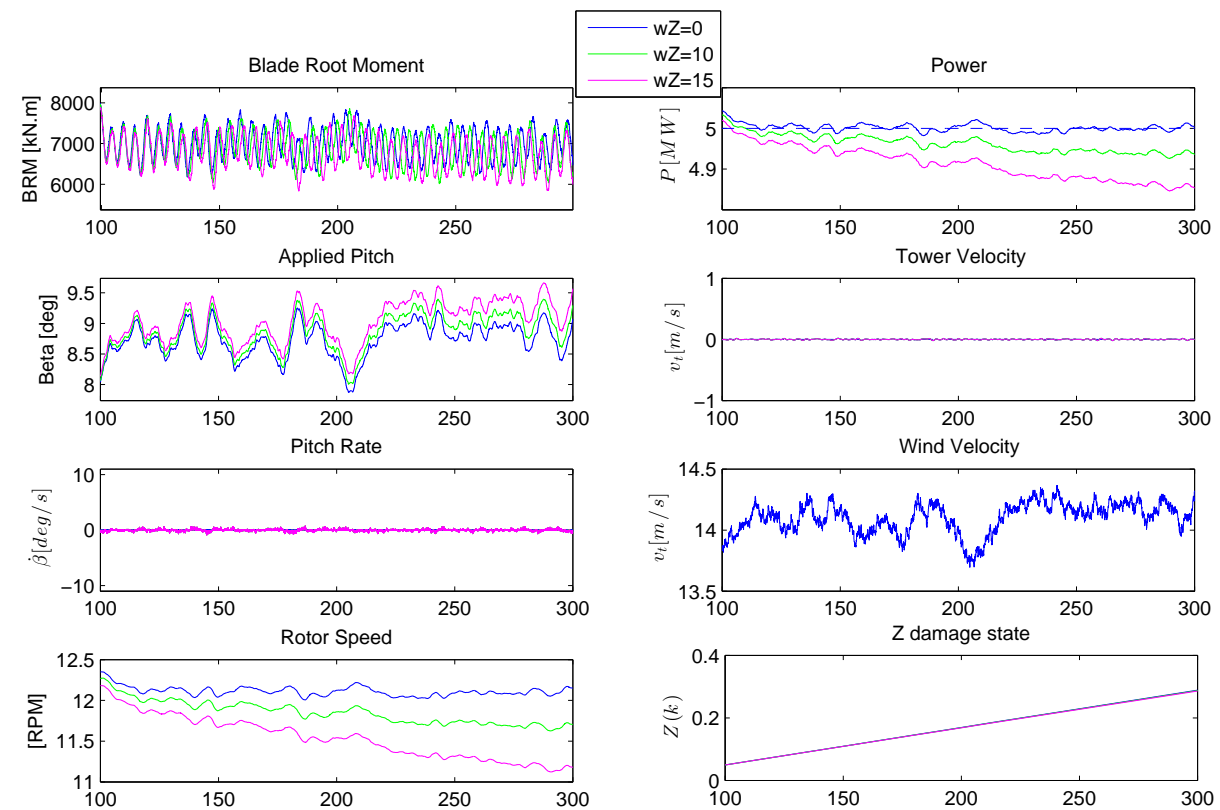

Figure 7. Evolution of the wind turbines variables and the accumulated damages for a turbulent wind of 14 $\mathrm{m} / \mathrm{s}$ mean speed
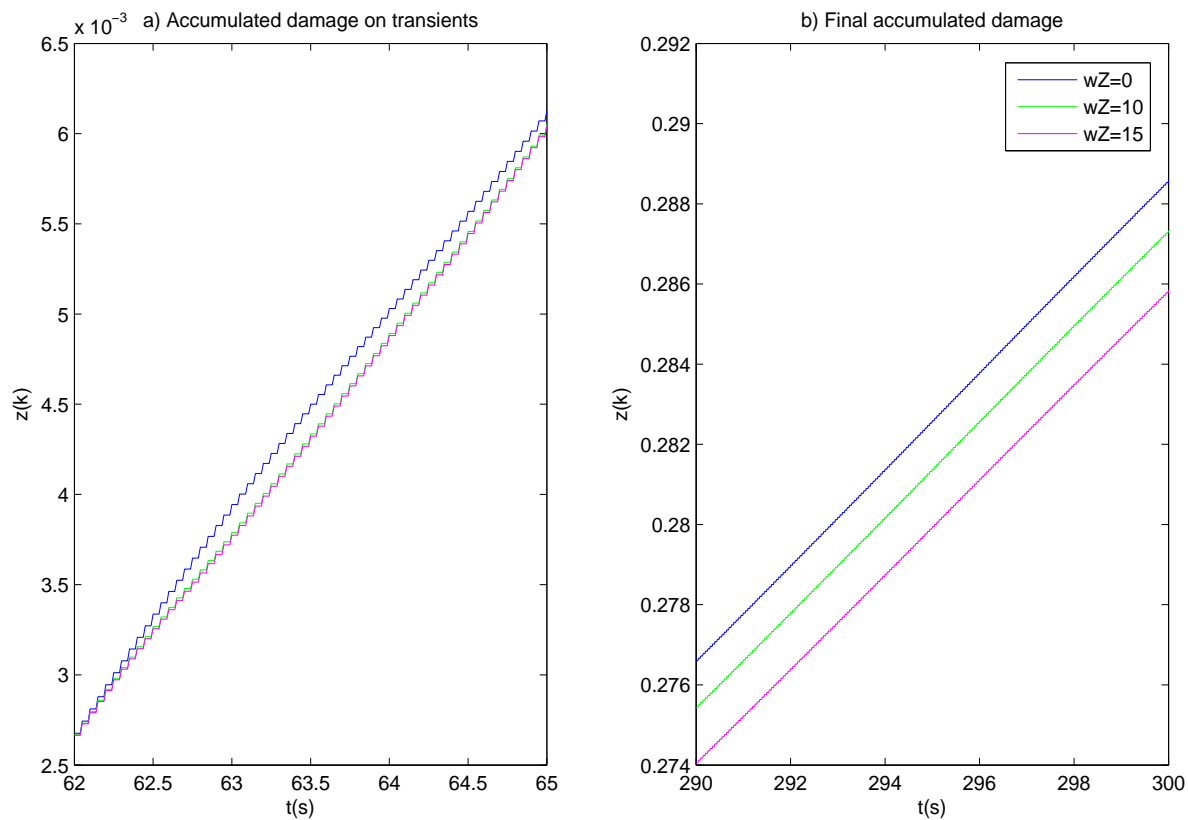

Figure 8. Detail of the accumulated damage of blade health state $z$ for a turbulent wind of $14 \mathrm{~m} / \mathrm{s}$ mean speed

\section{CONCLUSIONS}

The research presented in this paper has explored the integration of MPC with fatigue-based prognosis to minimize the damage of wind turbine components. The integration of a systems health 
Table I. Table showing the results of the accumulated damage and system performance variables for different weights of the MPC control for a turbulent wind with mean speed of $14 \mathrm{~m} / \mathrm{s}$.

\begin{tabular}{|c|c|c|c|c|}
\hline Weight $W_{z}$ & BRM mean (kN m) & Power (MW) & Rotor speed (rpm) & Accumulated Damage \\
\hline 0 & 7000.6 & 5 & 12.1 & 0.290 \\
\hline 10 & 6888.5 & 4.94 & 11.7 & 0.287 \\
\hline 15 & 6760.3 & 4.85 & 11.2 & 0.285 \\
\hline
\end{tabular}

Table II. Table showing the results of the accumulated damage and system performance variables for different weights of the MPC control for a turbulent wind with mean speed of $17 \mathrm{~m} / \mathrm{s}$.

\begin{tabular}{|c|c|c|c|c|}
\hline Weight $W_{z}$ & BRM mean (kN m) & Power (MW) & Rotor speed (rpm) & Accumulated Damage \\
\hline 0 & 5454 & 5 & 12.1 & 0.240 \\
\hline 10 & 5383.1 & 4.93 & 11.7 & 0.238 \\
\hline 15 & 5302.1 & 4.87 & 11.3 & 0.236 \\
\hline
\end{tabular}

management module with MPC control has provided the wind turbine with a mechanism to operate safely and optimize the trade-off between components life and energy production. The controller objective has been modified by adding an extra criterion that takes into account the accumulated damage. The scheme has been satisfactorily implemented and tested using a high fidelity simulator of a utility scale wind turbine. The results obtained show that there exists a trade-off between maximum power and the minimization of the accumulated damage. As future research, a way to find the optimal tuning of this trade-off will be investigated using multi-objective optimization techniques and the inclusion of a business model that assess the profitability in the trade-off that exists between power and fatigue reductions.

References

1. Vassilopoulos A. Fatigue life prediction of wind turbine blade composite materials. Advances in Wind Turbine Blade Design and Materials, Brøndsted P, Nijssen R (eds.). Woodhead Publishing, 2013; 251-297.

2. Musallam M, Johnson C. An efficient implementation of the rainflow counting algorithm for life consumption estimation. Reliability, IEEE Transactions on Dec 2012; 61(4):978-986.

3. Miner M. Cumulative damage in fatigue. Journal of Applied Mechanics 1945; 12(3):159-164.

4. Sutherland $\mathrm{H}$. On the fatigue analysis of wind turbines 1999.

5. Marín J, Barroso A, París F, Cañas J. Study of damage and repair of blades of a 300kw wind turbine. Energy 2008; 33(7):1068-1083.

6. Lampman S. Fatigue and Fracture, vol. 19. ASM International, 2009.

7. Gray C, Watson S. Physics of failure approach to wind turbine condition based maintenance. Wind Energy 2010; 13(5):395-405.

8. Frost $\mathrm{S}$, Goebel K, Obrecht L. Integrating structural health management with contingency control for wind turbines. International Journal of Prognostics and Health Management 2013; 4(9):11-20.

9. Lio WH, Rossiter J, Jones B. A review on applications of model predictive control to wind turbines. Control (CONTROL), 2014 UKACC International Conference on, 2014; 673-678.

10. Odgaaard P, Hovgaard T. On practical tuning of model uncertainty in wind turbine model predictive control. IFACPapersOnLine 2015; 48(30):327-332.

11. Odgaaard P, Hovgaard T. Selection of references in wind turbine model predictive control design. IFACPapersOnLine 2015; 48(30):333-338.

12. Odgaard P, Larsen L, Wisniewski R, Hovgaard T. On using pareto optimality to tune a linear model predictive controller for wind turbines. Renewable Energy 2016; 87:884-891. 
13. Adegas F, Wisniewski R, Sloth Larsen L. Gain-scheduled model predictive control of wind turbines using laguerre functions. American Control Conference (ACC), 2013, 2013; 653-658.

14. Soltani M, Wisniewski R, Brath P, Boyd S. Load reduction of wind turbines using receding horizon control. Control Applications (CCA), 2011 IEEE International Conference on, 2011; 852-857.

15. Henriksen L. Model predictive control of a wind turbine. PhD Thesis, DTU Technical University of Denmark, Kongens Lyngby, Denmark 2007.

16. Koerber A, King R. Combined feedback-feedward control of wind turbines using state-constraied model predictive control. IEEE Transactions on Control Systems Technology 2013; 21(4):1117-1128.

17. Evans M, Cannon M, Kouvaritakis B. Robust mpc tower damping for variable speed wind turbines. Control Systems Technology, IEEE Transactions on 2014; .

18. Dang D, Wang Y, Cai W. Nonlinear model predictive control (nmpc) of fixed pitch variable speed wind turbine. IEEE International Conference on Sustainable Energy Technologies, Singapore, 2008; 29-33.

19. Schlipf D, Schlipf D, Kühn M. Nonlinear model predictive control of wind turbines using LIDAR. Wind Energy 2013; 16(7):1107-1129.

20. Barradas-Berglind J, Wisniewski R, Soltani M. Fatigue damage estimation and data-based control for wind turbines. IET Control Theory and Applications 2015; 9(7):1042-1050.

21. Odgaard P, Knudsen T, Overgaard A, Steffensen H, Jørgensen M. Importance of dynamic inflow in model predictive control of wind turbines. 9th IFAC Symposium on Control of Power and Energy Systems CPES, New Delhi, India, 2015; 90-95.

22. Escobet T, Quevedo J, Puig V, Nejjari F. Combining health monitoring and control. Diagnostics and prognostics of engineering systems: methods and techniques, Kadry S (ed.). Engineering Science Reference. IGI Global, 2012; 230-256.

23. Pereira E, Bento R, Yoneyama T. Model predictive control using prognosis and health monitoring of actuators. Industrial Electronics (ISIE), 2010 IEEE International Symposium on, IEEE, 2010; 237-243.

24. Jonkman J, Marshall L. Fast User's Guide 2005.

25. Odgaard P, Johnson KE. Wind turbine fault detection and fault tolerant control - an enhanced benchmark challenge. Proceedings of the American Control Conference, pages 4447-4452 2013; .

26. Jonkman J. NREL offshore baseline 5MW wind turbine 2009.

27. Bianchi F, De Battista H, Mantz R. Wind Turbine Control Systems. Principles, Modelling and Gain Scheduling Design. Springer, 2007.

28. Esbensen T, Sloth C. Fault diagnosis and fault-tolerant control of wind turbines 2009.

29. Odgaard P, Stoustrup J, Kinnaert M. Fault-tolerant control of wind turbines: A benchmark model. IEEE Transactions on Control Systems Technology 2013; 21(4):1168-1182.

30. Maciejowski J. Predictive control with constraints. Prentice Hall: Essex, England, 2002.

31. Martinen S, Carlén I, Nilsson K, Breton SP, Ivanell S. Analysis of the effect of curtailment on power and fatigue loads of two aligned wind turbines using an actuator disc approach. Journal of Physics: Conference Series 2014; 524(1):012 182.

32. Endo T, Mitsunaga K, Nakagawa H. Fatigue of metals subjected to varying stress-prediction of fatigue lives. Preliminary Proceedings of the Chugoku-Shikoku District Meeting, 1967; 41-44.

33. Lee Y, Pan J, Hathaway R, Barkey M. Fatigue testing and analysis: theory and practice, vol. 13. ButterworthHeinemann, 2005.

34. Downing S, Socie D. Simple rainflow counting algorithms. International Journal of Fatigue 1982; 4(1):31-40.

35. Rychlik I. A new definition of the rainflow cycle counting method. International journal of fatigue $1987 ; \mathbf{9}(2): 119$ 121.

36. Niesłony A. Determination of fragments of multiaxial service loading strongly influencing the fatigue of machine components. Mechanical Systems and Signal Processing 2009; 23(8):2712-2721.

37. Hammerum K, Brath P, Poulsen N. A fatigue approach to wind turbine control. Journal of Physics: Conference Series, vol. 75, IOP Publishing, 2007; 012-081.

38. Jelavic M, Petrovic V, Peric N. Individual pitch control of wind turbine based on loads estimation. Industrial Electronics, 2008. IECON 2008. 34th Annual Conference of IEEE, 2008; 228-234.

39. Burton T, Jenkins N, Sharpe D, Bossanyi E. Wind Energy Handbook 2011.

40. Sanchez H, Escobet T, Puig V, Odgaard P. Fault diagnosis of advanced wind turbine benchmark using interval-based ARRs and observers. IEEE Transactions on Industrial Electronics 2015; 62(6):3783-3793.

41. Jonkman J, Butterfield S, Musial W, Scott G. Definition of a 5-MW Reference Wind Turbine for Offshore System Development 2009. 